profile was reported in only 37\%. Only 47\% ( $n=78$ ) of records described a witness account. Within the witness accounts that were recorded, key elements remained un-reported for example skin complexion was only reported in $35 \%$ of the 78 . The duration of the TLOC was recorded in only $44 \%$, Tongue biting in $27 \%$ and the presence or absence of abnormal movements was recorded in only $12 \%$ of this 78 patients. The presence or absence of a family history of sudden cardiac death was only reported in $2 \%$ cases. The family history of a cardiomyopathy was only recorded in $1 \%$ and a family history of TLOC was recorded in $1 \%$. A patient past history of cardiac disease was asked about in $40 \%$ of cases while a past history of TLOC was only asked about in $35 \%$. In this majority elderly study population, a recent change in drug therapy was only asked about in $2 \%$ of cases. This study highlights that in a DGH environment, the initial assessment of patients with TLOC is undertaken by junior medical staff who often do not document key diagnostically differentiating elements of the history and examination indicating an ongoing lack of adequate training regarding the most appropriate and accurate techniques for differentiating the causes of TLOC.

\section{AUTOMATED ANALYSIS OF ATRIAL ABLATION-SCAR USING DELAYED-ENHANCED CARDIAC MRI}

doi:10.1136/heartjnl-2011-300198.149

${ }^{1} \mathrm{~L}$ Malcolme-Lawes, ${ }^{1} \mathrm{R}$ Karim, ${ }^{2} \mathrm{C}$ Juli, ${ }^{2} \mathrm{P}$ B Lim, ${ }^{2} \mathrm{~T}$ V Salukhe, ${ }^{2} \mathrm{D}$ W Davies, ${ }^{1} \mathrm{D}$ Rueckert, ${ }^{1} \mathrm{~N}$ S Peters, ${ }^{2} \mathrm{P}$ Kanagaratnam. ${ }^{1}$ Imperial College London, London, UK; ${ }^{2}$ Imperial College Healthcare, London, UK

Introduction Visualisation of the ablation-related atrial scar using delayed-enhanced MRI (DE-MRI) may reveal important underlying causes for atrial fibrillation (AF) recurrence following ablation. In order to develop and objective method for delineating ablation-scar we compared pre and post DE-MRI after Cryo-balloon lesion on the basis that a more predictable lesion set would be created for validation.

Methods and Results 12 patients undergoing cryoablation for PAF were enrolled in the study, and underwent pre-ablation DE-MRI scans. Pulmonary vein isolation (PVI) was confirmed in all patients at the end of the cryoablation procedure using a circular mapping catheter. Additional ablation by RF or Freezer Max was required to achieve PVI in 59\%. No ablation was performed in any region other than the PV ostia. Post-ablation DE-MRI was performed at 3 months. An automatic segmentation of the LA was produced with custom software from the MRA sequence. The preablation and postablation free breathing late gadolinium enhanced sequence was registered to the MRA and the maximum intensity within the LA wall was projected onto the post ablation LA surface. The blood pool was identified automatically using custom software as the region $1 \mathrm{~cm}$ inside the wall of the LA, and its mean (BPM) and SD used as a baseline. To identify a universal threshold for scar, regions of brightest myocardium were initially selected in pre and post ablation MRIs. The brightest regions were $1.9 \pm 1.2$ vs $8.7 \pm 3.1$ SDs above the BPM in pre-and post-ablation MRIs respectively $(p=0.001)$. A threshold of 5 SDs above the BPM was therefore programmed into our custom software to identify regions of scar for all patients. The ostial regions were defined as extending $1 \mathrm{~cm}$ both proximal and distal to the PV-LA junction, and selected manually for left and right sided veins prior to scar projection. (See Abstract 149 figure 1). The scar proportion within these regions was calculated using commercially available software ITK-SNAP. Total LA scar proportion was $0.2 \pm 0.02 \%$ vs $6.3 \pm 0.75 \%$ in pre and post ablation scans respectively. The increase in scar seen in the PV ostia was $24.6 \pm 1.38 \%$ compared with $2.6 \pm 1.28 \%$ in the rest of the LA $(\mathrm{p}=0.01)$ (See Abstract 149 figure 2).

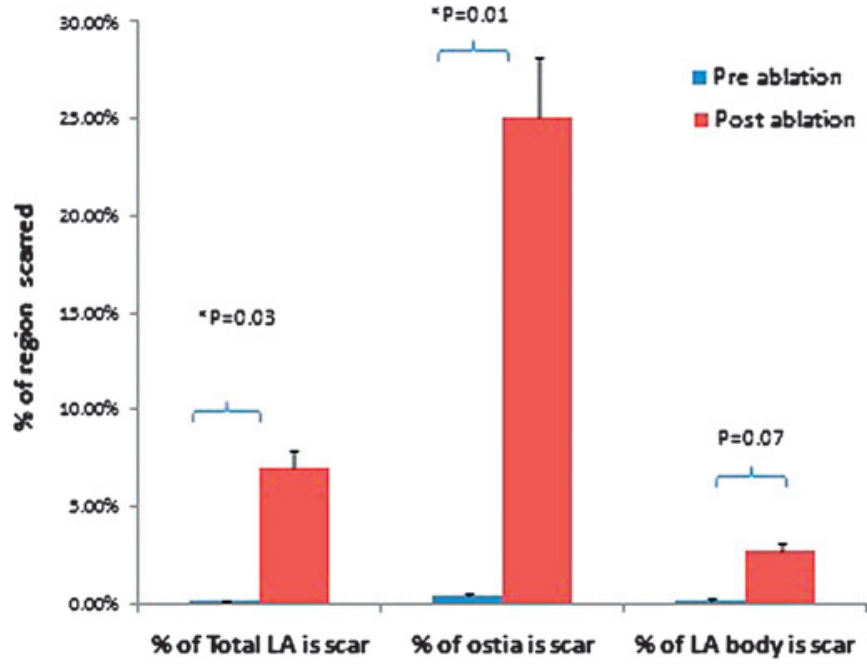

Abstract 149 Figure 1 Comparison of pre-ablation and post-ablation \% scar using fixed threshold.
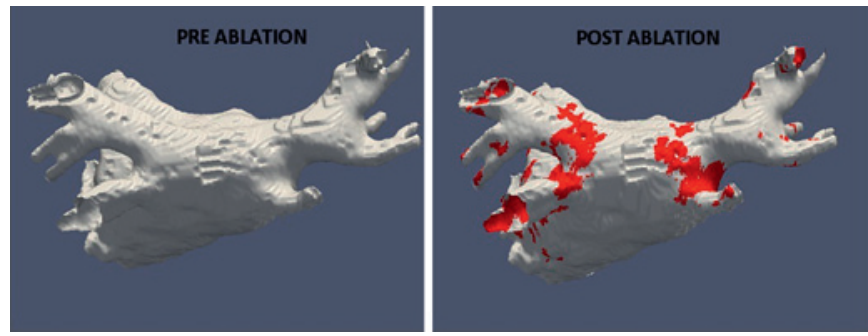

Abstract 149 Figure 2

Conclusion We have demonstrated the feasibility an objective, automated method of DE-MRI analysis of left atrial ablation-scar. This technique will now need to be validated against clinical outcomes.

\section{IMPLANTABLE CARDIOVERTER-DEFIBRILLATOR LEAD COMPLICATIONS AND CLINICAL EFFECTIVENESS IN PATIENTS WITH INHERITED CARDIAC CONDITIONS}

doi:10.1136/heartjnl-2011-300198.150

${ }^{1,2} \mathrm{R}$ Bastiaenen, ${ }^{1} \mathrm{~S}$ Ben-Nathan, ${ }^{2} \mathrm{~S}$ Jones, ${ }^{2} \mathrm{D}$ Ward, ${ }^{2} \mathrm{M}$ Gallagher, ${ }^{1,2} \mathrm{~S}$ Sharma, ${ }^{1,2} \mathrm{E}$ R Behr. ${ }^{1}$ St George's University of London, London, UK; ${ }^{2}$ St George's Hospital, London, UK

Background Implantable cardioverter-defibrillator (ICD) therapy can reduce sudden death due to ventricular arrhythmia (VT/VF) but is not without complication, particularly in young patients who live for many years with a device in situ. We aimed to determine the ICD complication rate in our inherited cardiac condition (ICC) population compared with international reports. Particular importance was given to inappropriate shock therapy due to lead failure as there are new ICD technologies available.

Methods Patients with ICCs who had ICD implantation or box change between January 2006 and September 2009 were included. Data on clinical characteristics, complications and ICD therapies were obtained from pacing and hospital records. We compared our data with several ICD studies of patients with specific ICCs (Abstract 150 table 1). 